\title{
Learning Approximate MRFs from Large Transaction Data ${ }^{\star}$
}

\author{
Chao Wang and Srinivasan Parthasarathy \\ Department of Computer Science and Engineering, The Ohio State University \\ srini@cse.ohio-state.edu
}

\begin{abstract}
In this paper we consider the problem of learning approximate Markov Random Fields (MRFs) from large transaction data. We rely on frequent itemsets to learn MRFs on the data. Since learning exact large MRFs is generally intractable, we resort to learning approximate MRFs. Our proposed modeling approach first employs graph partitioning to cluster variables into balanced disjoint partitions, and then augments important interactions across partitions to capture interdependencies across them. A novel treewidth based augmentation scheme is proposed to boost performance. We learn an exact local MRF for each partition and then combine all the local MRFs together to derive a global model of the data. A greedy approximate inference scheme is developed on this global model. We demonstrate the use of the learned MRFs on the selectivity estimation problem. Empirical evaluation on real datasets demonstrates the advantage of our approach over extant solutions.
\end{abstract}

\section{Introduction}

In this paper we address the problem of learning approximate Markov Random Fields (MRF) from large transaction data. Examples of such data include market basket data, web log data, etc. Such data can be represented by a high-dimensional data matrix, with each row corresponding to a particular market basket (web session), and each column corresponding to a particular item (web page). Each entry takes a value of " 1 " if the corresponding item is in the corresponding basket, otherwise it takes a value of " 0 ". The data matrix is binary, and very often in such applications, highly sparse in that the number of non-zero entries is small.

To model such data effectively in order to answer queries about the data efficiently, we consider the use of probabilistic models. Probabilistic models capture association or causal correlations among attributes in data and have been successfully applied in applications such as selectivity estimation in query optimization [1.23], link analysis/recommender systems [4]5] and bioinformatics [6].

Specifically to tackle the selectivity estimation problem, Pavlov et al. [3] propose a Maximum Entropy (ME) model based on frequent itemsets. The ME model is essentially equivalent to an MRF and is effective in estimating query selectivity. However, a key limitation of their approach is that it needs to learn a local model over query variables

\footnotetext{
* This work is supported in part by the following research grants: DOE Award No. DE-FG0204ER25611; NSF CAREER Grant IIS-0347662.
} 
on the fly for every query. Due to the fact that inferring an ME model is an expensive iterative process, such a just-in-time model construction approach is not appropriate in settings where online estimation time is crucial. The alternative is to first learn a global model offline. Subsequently the queries can be answered on the fly using standard probabilistic inference methods [789]. The advantages are a more accurate model (relies on complete information from all the data) and online performance. The critical challenge is that a global model may be prohibitive to compute for large datasets of high dimension (offline learning cost) . To address this problem, in this paper, we consider the problem of employing frequent itemsets to learn approximate global MRFs on large transaction data. Frequent itemsets capture important local distribution information of the data. Hollmen et al. [10] proposed to use frequent itemsets to learn mixture models on the local scale. Goldenberg et al. [5] proposed an approach (SNBS) of using frequent itemsets to learn large Bayesian networks.

The main contributions of this paper are summarized below: a. We introduce a novel divide-and-conquer style approach based on graph partitioning to learning approximate MRFs from large transaction data; $\mathbf{b}$. We introduce a novel interaction importance and treewidth based augmentation scheme to capture interdependencies across partitions; c. We conduct an extensive empirical study on real datasets to show the efficiency and effectiveness of the new approach.

\section{Background}

Let $\mathcal{I}$ be a set of items, $i_{1}, i_{2}, \ldots, i_{d}$. A subset of $\mathcal{I}$ is called an itemset. The size of an itemset is the number of items it contains. An itemset of size $k$ is a $k$-itemset. A transaction dataset is a collection of itemsets, $D=\left\{t_{1}, t_{2}, \ldots, t_{n}\right\}$, where $t_{i} \subseteq \mathcal{I}$. For any itemset $\alpha$, we write the transactions that contain $\alpha$ as $D_{\alpha}=\left\{t_{i} \mid \alpha \subseteq t_{i}\right.$ and $t_{i} \in$ $D\}$. In the probabilistic model context, each item is modeled as a random variable 1 .

Definition 1. (Frequent itemset). For a transaction dataset $D$, an itemset $\alpha$ is frequent if $\left|D_{\alpha}\right| \geq \sigma$, where $\left|D_{\alpha}\right|$ is called the support of $\alpha$ in $D$, and $\sigma$ is a user-specified non-negative threshold.

Definition 2. (Markov Random Field). An Markov Random Field (MRF) is an undirected graphical model in which vertices represent variables and edges represent correlations between variables. The joint distribution associated with an undirected graphical model can be factorized as follows: $p(X)=\frac{1}{Z(\psi)} \prod_{C_{i} \in \mathcal{C}} \psi_{C_{i}}\left(X_{C_{i}}\right)$, where $\mathcal{C}$ is the set of maximal cliques associated with the undirected graph; $\psi_{C i}$ is a potential function over the variables of clique $C_{i}$ and $\frac{1}{Z(\psi)}$ is a normalization term.

Using Frequent Itemsets to Learn an MRF. The idea of using frequent itemsets to learn an MRF was first proposed by Pavlov et al. [3]. A $k$-itemset and its support represents a $k$-way statistic and can be viewed as a constraint on the true underlying distribution that generates the data. Given a set of itemset constraints, a Maximum Entropy (ME) distribution satisfying all these constraints is selected as the estimate for the true

\footnotetext{
${ }^{1}$ In this article we use these terms - item, (random) variable - interchangeably.
} 


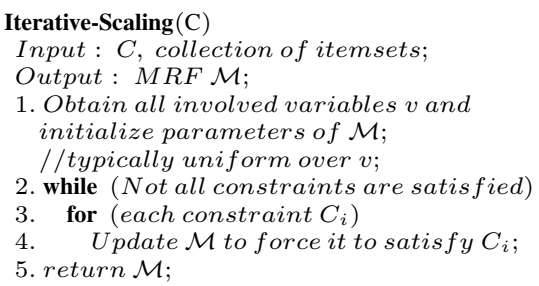

Fig. 1. Iterative scaling algorithm

underlying distribution. This ME distribution is essentially equivalent to an MRF. A simple iterative scaling algorithm can be used to learn an MRF from a set of itemsets. Figure 1 presents a high-level outline of a computationally efficient version of the algorithm given by Jelinek [11]. Efficient inference is crucial to the running time of the learning algorithm. We call models learned through exact inference procedures exact.

The junction tree algorithm is a commonly-used exact inference engine for probabilistic models. The time complexity of the junction tree algorithm is exponential in the treewidth of the underlying model. For real world models, it's quite common that the treewidth will be well above 20, making learning exact models intractable. As a result, we have to resort to learning approximate models. One possible approach is to plug in approximate inference engines during the model learning process. However, it is not clear whether or not the learning process will still converge when subjected to approximate inference engines. In this paper, we pursue another approach - to learn a simplified model which is feasible to learn exactly and is close to the true exact model.

\section{Learning Approximate MRFs}

Before discussing our proposed approach, let us consider an extreme case in which the overall graph consists of a set of disjoint non-correlated components. Then the joint distribution can be obtained in a straightforward fashion according to Lemma 1 .

Lemma 1. 2 Given an undirected graph $G$ subdivided into disjoint components $D_{1}$, $D_{2}, \ldots, D_{n}$ (not necessarily connected components), and there is no edge across any two components, then the probability distribution associated with $G$ is given by: $p(X)=$ $\prod_{i=1}^{n} p\left(X_{D i}\right)$

\subsection{Clustering Variables Based on Graph Partitioning}

The basic idea of our proposed divide-and-conquer style approach comes directly from the above observation. Specifically, the variables are clustered into groups according to their correlation strengths. We call the group variable-cluster. Then a local MRF is defined on each variable-cluster. In the end we aggregate the local models to obtain a global model. From Lemma1, we see that if we have a perfect partitioning of an MRF

\footnotetext{
${ }^{2}$ This follows immediately from the global Markov property of the MRF.
} 
in which there is no correlations across different partitions, the divide-and-conquer style approach gives the exact estimate of the full model. Even for an imperfect partitioning, if the correlations across partitions are not strong, we still expect a reasonable approximation of the full model. Correspondingly, the first problem we face is how to cluster the variables such that the correlations across partitions is minimized.

$k$-MinCut. The $k$-MinCut problem is defined as follows [12]: Given a graph $G=$ $(V, E)$ with $|V|=n$, partition $V$ into $k$ subsets, $V_{1}, V_{2}, \ldots, V_{k}$ such that $V_{i} \cap V_{j}=\emptyset$ for $i \neq j,\left|V_{i}\right|=\frac{n}{k}$, and $\cup_{i} V_{i}=V$, and the number of edges of $E$ whose incident vertices belong to different subsets is minimized. Given a partitioning $P$, the number of edges whose incident vertices belong to different partitions is called the edge-cut of the partitioning. In the case of weighted graphs, we minimize the sum of weights of all edges across different partitions.

The $k$-MinCut can serve our purpose of clustering variables. Each graph partition corresponds to a variable-cluster. Intuitively, we want to maximize correlations among variables within variable-clusters, and minimize correlations among variables across variable-clusters. So we should make the weight of edges reflect the strength of correlations between variables. We have the collection of all frequent itemsets. In particular, itemsets of size 2 specify the connectedness structure of the graph, and their associated supports indicate the strength of pairwise correlations between variables. We can use their supports as the edge weights directly. However, we also have higher-order statistics available, i.e., the larger itemsets. We expect that taking into consideration the information of all itemsets will yield a better weighting scheme. To this end, we propose an accumulative weighting scheme as follows: for each itemset, we add its support to all related edges, whose two vertices are contained by the itemset. Intuitively, we strengthen the graph regions which involve closely related itemsets in the hope that the edges within these regions will not be broken in the partitioning. An advantage of the $k$-MinCut partitioning scheme is that the resulting clustering is forced to be balanced. This is desirable for the sake of efficient model learning, since we will not encounter very large variable-clusters which might result in very complex local models.

\subsection{Interaction Importance and Treewidth Based Variable-Cluster Augmentation}

The balanced variable-clusters produced by the $k$-MinCut partitioning scheme are disjoint. Intuitively, there is significant correlation information that is lost during the partitioning. To compensate for this loss, we propose an interaction importance based variable-cluster augmenting scheme to recover the damaged correlation information. The idea is that for each variable-cluster, we let it grow outward. More specifically, it attracts and absorbs most significant (important) interactions (edges) incident to its vertices from outside to itself. As a result, some extra variables are pulled into the variable-cluster. We control the augmentation through the number of extra vertices pulled into the cluster (called growth factor). One can use the same growth factor for all variable-clusters to preserve their balance.

As an optimization, we account for the model complexity during the augmentation. We keep augmenting a partition until its complexity reaches a user-specified threshold. 
More specifically, we keep track of the growth of the treewidth during the augmenting process for this purpose. 1-hop neighboring vertices are first considered by the augmentation, followed by 2-hop neighboring vertices and so on. Meanwhile, we still stick to the interaction importance criteria. The resultant augmented partitions are likely to become unbalanced in terms of their size. The partitions with a small treewidth will grow more significantly than those with a large treewidth. However, these partitions are balanced in terms of their complexity. A benefit of this scheme is that usually more interactions across different partitions will be accounted for in a computationally controllable manner, leading to a more accurate global model. Figure ?? presents a sketch of the overlapped variable-clusters after the augmentation.

\subsection{Approximate Global MRFs and a Greedy Inference Algorithm}

For each augmented variable-cluster, we collect all of its related itemsets and use the iterative scaling algorithm to learn an exact local model. This is computationally feasible since the local model corresponding to each variable-cluster is much simpler than the original model. Two local models are correlated to each other if they share variables. The collection of all local models forms a global model of the original transaction data. We note that this global model is an approximation of the exact global MRF, since we lose dependency information by breaking edges in the exact graphical model. However, most strong correlations are compensated during the variable-cluster augmentation. As such, we believe that the proposed global model reasonably approximates the exact model. Figure 2 provides the formal algorithm for learning an approximate global MRF.

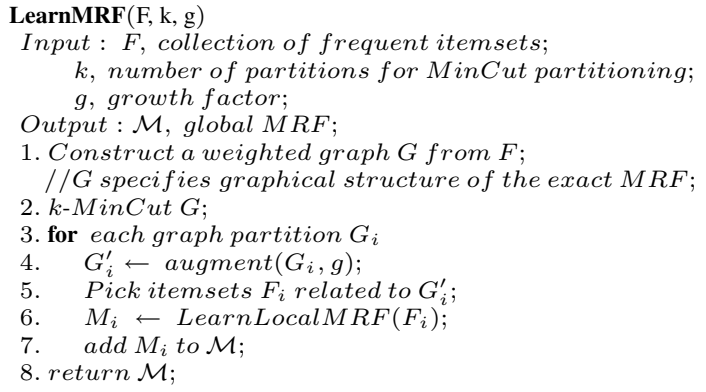

Fig. 2. Learning approximate global MRF algorithm

Given the global model consisting of a set of local MRFs, how do we make inferences on this model efficiently? In the first case, where all query variables are subsumed by a single local MRF, we just need to calculate the marginal probability within the local model. In the second case, where query variables span multiple local models, we use a greedy decomposition scheme to compute. First, we pick the local model that has the largest intersection with the current query (i.e., covers most query variables). Then we pick the next local model that covers most uncovered variables in the query. This covering process will be repeated until we cover all variables in the query. Simultaneously, 
all intersections between the above local models and the query are recorded. In the end, we derive an overlapped decomposition of the query. We notice that locally the dependency among small pieces in the decomposition often exhibits a tree-like structure, and we use Lemma2 to compute the marginal probabilities.

Lemma 2. 3 Given an undirected graph $G$ subdivided into $n$ overlapped components, if there exists an enumeration of these $n$ components, i.e., $C_{1}, C_{2}, \ldots, C_{n}$, s.t., for any $2 \leq i \leq n$, the separating set, $s\left(C_{i}, \cup_{j=1}^{i-1} C_{j}\right) \subseteq\left(C_{i} \cap\left(\cup_{j=1}^{i-1} C_{j}\right)\right)$, then the probability distribution associated with $G$ is given by: $p(X)=\frac{\prod_{i=1}^{n} p\left(X_{C_{i}}\right)}{\prod_{i=2}^{n}{ }^{p\left(X_{C_{i}} \cap\left(\cup_{j=1}^{i-1} X_{C_{j}}\right)\right)}}$

Essentially, Lemma2 2 specifies a junction tree-like structure. Given any model and one of its such decomposition, we can use the above formula to make exact inferences. However, it is possible to have cyclic dependencies among the decomposed pieces. Therefore, the greedy inference scheme is a heuristic. Also, we note that our global model is not globally consistent in that there exists inconsistency across the local models. However, we expect that the global model is nearly consistent since two correlated local models support the same evidence (itemsets) regarding their shared variables.

\section{Experimental Results}

In this section, we examine the performance of our proposed approach on real datasets. We focus on its application on the selectivity estimation problem. We compare our proposed model against the previous approach in [3] where a local MRF over query variables is learned for every query in an online fashion. We call this approach online local MRF approach (abbreviated as $O L M$ ).

Experimental Setup: All the experiments were conducted on a Pentium $42.66 \mathrm{GHz}$ machine with 1 GB RAM running Linux 2.6.8. The MRF learning algorithm was implemented in C++. We used apriori [14] to collect frequent itemsets and Metis [12] to obtain a $k$-MinCut of the exact graphical model.

Datasets: We used two publicly available datasets in our experiments: the Microsoft Anonymous Web dataset (kdd.ics.uci.edu) with 32711 transactions and 294 items; the BMS-Webview1 dataset (fimi.cs.helsinki.fi) with 59602 transactions and 497 items. Query Workloads: We considered the workloads consisting of conjunctive queries of different sizes. Following the same practice in [3], we first specified the number of query variables $n$ (varied from $4,6,8,10$ to 12 ), then we picked $n$ variables according to the probability of the variable taking a value of " 1 " and generated a value for each selected variable by its univariate probability distribution. Performance Metrics: We considered the online time cost, the time taken to answer the queries using the model. We also considered the offline time cost, the time taken to learn the model. We quantified the accuracy of estimations using the average absolute relative error over all queries in the workload. The absolute relative error is defined as $|\sigma-\hat{\sigma}| / \sigma$, where $\sigma$ is the true selectivity and $\hat{\sigma}$ is the estimated selectivity.

${ }^{3}$ The complete proof can be found in the full version of this paper [13]. 
Results on the Microsoft Web Data. In this section, we report the experimental results on the Microsoft Web Data. We use the support threshold of 20 to collect the frequent itemsets, which results in 9901 frequent itemsets. According to the Maximum Cardinality Search (MCS)-ordering heuristic [15], the treewidth of the resulting MRF is 28 for which learning the exact model is intractable.
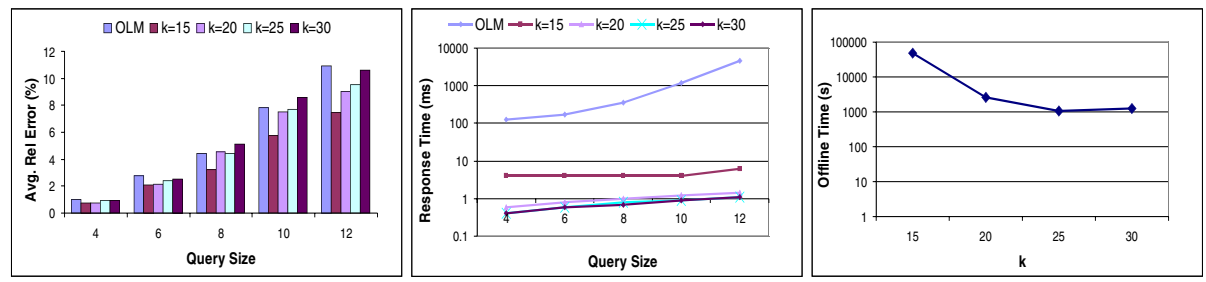

Fig. 3. Varying $k(g=5)$ : (a) estimation accuracy (b) online time (c) offline time

Figure 3a presents the estimation accuracy when $k$ is varied ( $g$ is fixed as 5 ) for queries of different sizes. As seen, our approach works very well compared with the online local MRF approach. Our approach gives very close or even better estimations compared with the online local MRF approach. These results are not surprising since for the online local model, we only use the local information to estimate the selectivity. However, for the offline global model, we rely on the global information to make the estimation. Even though the graph partitioning phase results in information loss, since the model is global in nature, in many cases it is still able to yield better estimations. Furthermore, an obvious trend that stands out is that as the query size increases, the quality of the estimations degrades. This is expected since for larger sized queries, estimation errors grow for both approaches. Another observation is that the estimations are more accurate when we use less variable-clusters. This is because with less variableclusters, the information loss due to the graph partitioning is smaller, thus we capture better the correlations between partitions. Figure $3 \mathrm{~b}$ illustrates how the online times depend on $k$. It can be clearly seen the significant growth of the online times taken by the online model (note the Y-axis scale). The extreme online timing efficiency of the offline model can be clearly seen from the results. In most cases, it outperformed the online model by two to three orders of magnitude. Further, we see that the smaller $k$ results in higher online estimation time. This is expected since the smaller $k$ is, the larger each local model will be, which explains the slower estimation. In the extreme case where $k$ is 1 , we revert to learning the exact global MRF, which has been shown to be computationally infeasible. Figure $3 \mathrm{k}$ presents the offline learning times of the offline model when varying $k$. An obvious trend is that as we increase $k$, overall the learning cost of the offline model decreases significantly. This is expected since the larger $k$ results in less complex local models.

Figure 4h presents the estimation accuracy when varying $g$ ( $k$ is fixed as 20). As one can see, the error decreases steadily with increasing $g$. When $g$ is 0 (disjoint variableclusters), the estimations are most inaccurate. In contrast, the estimations are much 

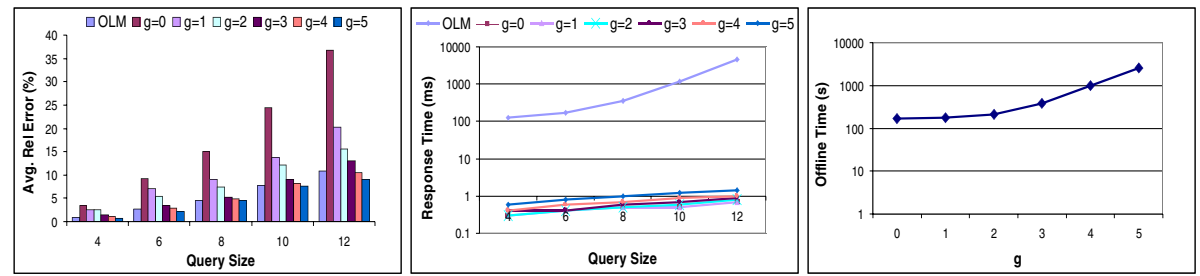

Fig. 4. Varying $g(k=20)$ : (a) estimation accuracy (b) online time (c) offline time

more accurate when $g$ is 5 . The results clearly show the effects of the interaction importance based variable-cluster augmenting scheme. The offline model approximates the exact global model better when more correlations across the local models are compensated. Figure $4 \mathrm{~b}$ presents the online times when varying $g$. We see from the results that the model with the larger $g$ takes more online time to answer the query. This is also expected since the larger $g$ results in more complex models (similar to the case of the smaller $k$ ). Figure $4 k$ presents the offline learning times of the offline model when varying $g$. An obvious trend is that as we increase $g$, the time cost increases significantly, which is again expected.
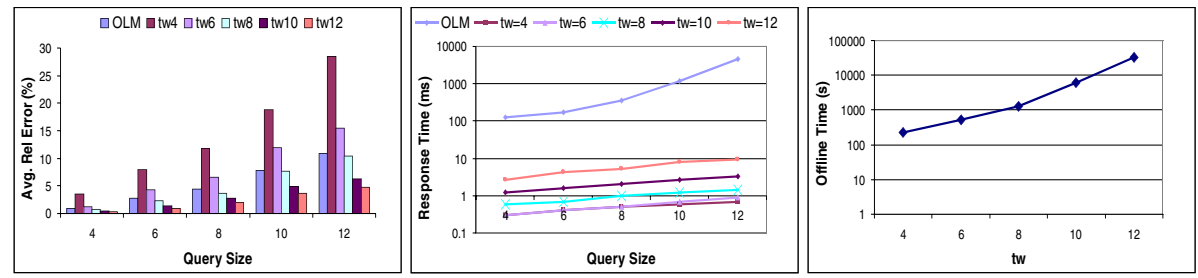

Fig. 5. Varying $t w(k=25)$ : (a) estimation accuracy (b) online time (c) offline time

Figure 5a-c present the estimation accuracy, the online times and the offline learning times of the offline global model when the treewidth based augmentation optimization is used ( $k$ is fixed as 25). As seen, the optimization can further boost the estimation performance. For example, the average relative estimation errors are $0.29 \%, 0.97 \%$, $2.01 \%, 3.66 \%$ and $4.81 \%$ on the workloads consisting of queries of size $4,6,8,10$ and 12 , respectively. In contrast, the corresponding errors of the online local MRF approach are $0.99 \%, 2.76 \%, 4.45 \%, 7.82 \%$ and $10.9 \%$, respectively. Furthermore, the offline model is faster by about two orders of magnitude in terms of online estimating time. Moreover, as we raise the treewidth threshold, the estimations will become more accurate, at a higher cost of online estimating and offline learning times.

The results on the BMS-Webview1 dataset overall are quite similar to that on the Microsoft Web dataset and are omitted in the interest of space. However, the complete results can be found in [13]. 


\section{Conclusion}

In this paper, we have described a new approach to learning an approximate MRF on large transaction data. Our proposed approach has been shown to be very effective and efficient in solving the selectivity estimation problem. In the future, we would like to exploit a belief propagation style approach to force the consistency of the model. Furthermore, we would like to investigate the use of the approximate inference techniques during the model learning process. Finally, it would be interesting to exploit the learned models on various link analysis tasks.

\section{References}

1. Getoor, L., Taskar, B., Koller, D.: Selectivity estimation using probabilistic models. In: SIGMOD Conference 2001. (2001) 461-472

2. Deshpande, A., Garofalakis, M.N., Rastogi, R.: Independence is good: Dependency-based histogram synopses for high-dimensional data. In: SIGMOD Conference 2001. (2001) 199-210

3. Pavlov, D., Mannila, H., Smyth, P.: Beyond independence: probabilistic models for query approximation on binary transaction data. IEEE Transactions on Knowledge and Data Engineering 15 (2003) 1409-1421

4. Breese, J.S., Heckerman, D., Kadie, C.M.: Empirical analysis of predictive algorithms for collaborative filtering. In: Proceedings of the Fourteenth Conference on Uncertainty in Artificial Intelligence. (1998) 43-52

5. Goldenberg, A., Moore, A.: Tractable learning of large bayes net structures from sparse data. In: Proceedings of the twenty-first international conference on Machine learning. (2004)

6. Friedman, N.: Inferring cellular networks using probabilistic graphical models. Science $\mathbf{3 0 3}$ (2004) 799-805

7. Lauritzen, S., Speigelhalter, D.: Local computations with probabilities on graphical structures and their application to expert systems. Journal of the Royal Statistical Society, Series B (Methodological) 50 (1988) 157224

8. Jordan, M.I., Kearns, M.J., Solla, S.A.: An introduction to variational methods for graphical models. Machine Learning 37 (1999) 183-233

9. Yedidia, J.S., Freeman, W.T., Weiss, Y.: Understanding belief propagation and its generalizations. In: IJCAI. (2001)

10. Hollmen, J., Seppanen, J.K., Mannila, H.: Mixture models and frequent sets: Combining global and local methods for 0-1 data. In: Proceedings of the Third SIAM International Conference on Data Mining. (2003)

11. Jelinek, F.: Statistical Methods for Speech Recognition. MIT Press, Cambridge, MA (1998)

12. Karypis, G., Kumar, V.: Multilevel k-way partitioning scheme for irregular graphs. J. Parallel Distrib. Comput. 48 (1998) 96-129

13. Wang, C., Parthasarathy, S.: Learning approximate mrfs from large transaction data. In: The Ohio State University, Technical Report. (2006)

14. Agrawal, R., Srikant, R.: Fast algorithms for mining association rules in large databases. In: Proceedings of the 20th International Conference on Very Large Data Bases. (1994) 487-499

15. Tarjan, R.E., Yannakakis, M.: Simple linear-time algorithms to test chordality of graphs, test acyclicity of hypergraphs, and selectively reduce acyclic hypergraphs. SIAM Journal of Computing 13 (1984) 